\title{
Characterization and Directional Visualization of Space Radiation Quanta in Low Earth Orbit with the compact Spacecraft Payload SATRAM
}

\author{
Carlos Granja1, Stepan Polansky, Stanislav Pospisil, Zdenek Vykydal, Daniel \\ Turecek \\ Institute of Experimental and Applied Physics (IEAP), Czech Technical University in Prague (CTU) \\ Horska 3a/22, 12800 Prague 2, Czech Republic \\ E-mail: carlos.granja@utef.cvut.cz
}

\author{
Alan Owens, Karim Mellab, Petteri Nieminen \\ European Space Research \& Technology Centre (ESTEC), European Space Agency (ESA) \\ Postbus 299, NL-2200 AG Noordwijk, The Netherlands
}

\author{
Zdenek Dvorak, Marek Simcak, Zdenek Kozacek", Petr Vana", Jan Mares\# \\ Czech Space Research Center (CSRC) \\ Janska 12, 60200 Brno, Czech Republic
}

\begin{abstract}
In this contribution the response of the SATRAM/Timepix spacecraft payload to space radiation in Low Earth Orbit in the form of full Timepix frames along a single satellite orbit is presented. The compact light weight SATRAM payload has been operating in open space onboard ESA's Proba-V satellite in low Earth orbit since $7^{\text {th }}$ May 2013. The embedded Timepix chip can determine the composition and spectral characteristics of ionizing radiation in the satellite environment together with visualization of single particle tracks. The device provides single quantum X-ray photon and charged particle counting for high sensitivity detection, high resolution tracking and directional visualization of energetic charged particles over a wide dynamic range of particle fluxes, energies and broad field of view. In addition to the visualization of the varying radiation environment along a single satellite orbit also preliminary evaluated results in the form of total particle flux maps (navigation correlated spatial and time distributions) along the satellite orbit for a period of 19 days in October 2014 are included.
\end{abstract}

The 23rd International Workshop on Vertex Detectors

15-19 September 2014

Macha Lake, The Czech Republic

\footnotetext{
${ }^{1}$ Speaker; ${ }^{*}$ former staff
} 


\section{Introduction}

The Space Application of Timepix Radiation Monitor (SATRAM) is a compact low-mass and low-power spacecraft payload operating in open space on board in the ESA's Proba-V satellite [1]. Launched on $7^{\text {th }}$ May 2013, the satellite is deployed in Low Earth Orbit (LEO) at an altitude $820 \mathrm{~km}$ (sun synchronous polar orbit tilted at $98.8^{\circ}$ ). SATRAM provides highsensitivity wide-dynamic range radiation monitoring enhanced with particle track visualization of the charged events and low-energy X-ray field along the satellite orbit. The Timepix detector [2] determines over a wide range of particle fluxes, energies and broad field of view [3] the composition (particle species) and spectral characterization $(\mathrm{dE} / \mathrm{dx})$ of the mixed field radiation environment [4]. The per-pixel energy sensitivity provides linear energy transfer (LET) spectra with enhanced particle-type resolving power and directional sensitivity for energetic charged particles. Results include spatial- and time-dependent distributions of the radiation environment along the satellite orbit.

The motivation for this work and a more detailed description of the payload instrument (design, operation, response) have been presented [5] where preliminary results in the form spatial Earth maps of total radiation dose rate were included. In this contribution a short description of the core detector technology (Timepix chip) and of the spacecraft payload (SATRAM) is given. Results presented are in the form of visualization of the mixed radiation field in the environment of the satellite along a single orbit showing the full frames of the embedded Timepix detector at selected navigation stamps. In addition, the preliminary maps (spatial, time distributions) of total particle flux measured along the satellite orbit for a period of 19 days collected in October 2014 are included.

\section{The hybrid semiconductor pixel detector Timepix}

The position-sensitive semiconductor detectors of the Medipix family [2,6], developed in frame of the MEDIPIX Collaboration provide high sensitivity (single-particle/quantum counting), wide-dynamic range, high spatial resolution and noiseless (dark-current free) detection. The hybrid architecture enables using sensors of defined media (e.g., Si, CdTe, GaAs) and thicknesses (e.g., $300 \mathrm{um}, 700 \mathrm{um}, 1000$ and $1500 \mathrm{um}$ ). The detector consists of a matrix of $256 \times 256$ (total of 65.536) pixels, pixel pitch size $55 \mu \mathrm{m}$ and full sensitive area $14 \mathrm{~mm} \times 14$ $\mathrm{mm}\left(1.98 \mathrm{~cm}^{2}\right)$ - see Fig. 1 .

Based on the Medipix2 [6] device, Timepix [2] provides extended functionality at the level of the per-pixel integrated electronic chain. Each pixel of the Timepix detector can be independently configured to operate in counting mode (the counter is incremented by one when the per-pixel deposited energy of the interacting particle crosses a threshold level), energy mode (called Time-over-Threshold (ToT) mode where the counter is incremented continuously as long as the signal is above threshold) and Time mode (called Time-of-Arrival (ToA) mode where the counter is incremented continuously from the time the first hit arrives until the closure of the shutter or end of the time window or acquisition exposure). Equipped with a 300 $\mu \mathrm{m}$ thick silicon sensor, Timepix is sensitive to X-rays (highest efficiency in the range 5-25 $\mathrm{keV}$ ) and charged particles (100\% detection efficiency) with a detection threshold of $4 \mathrm{keV}$. 

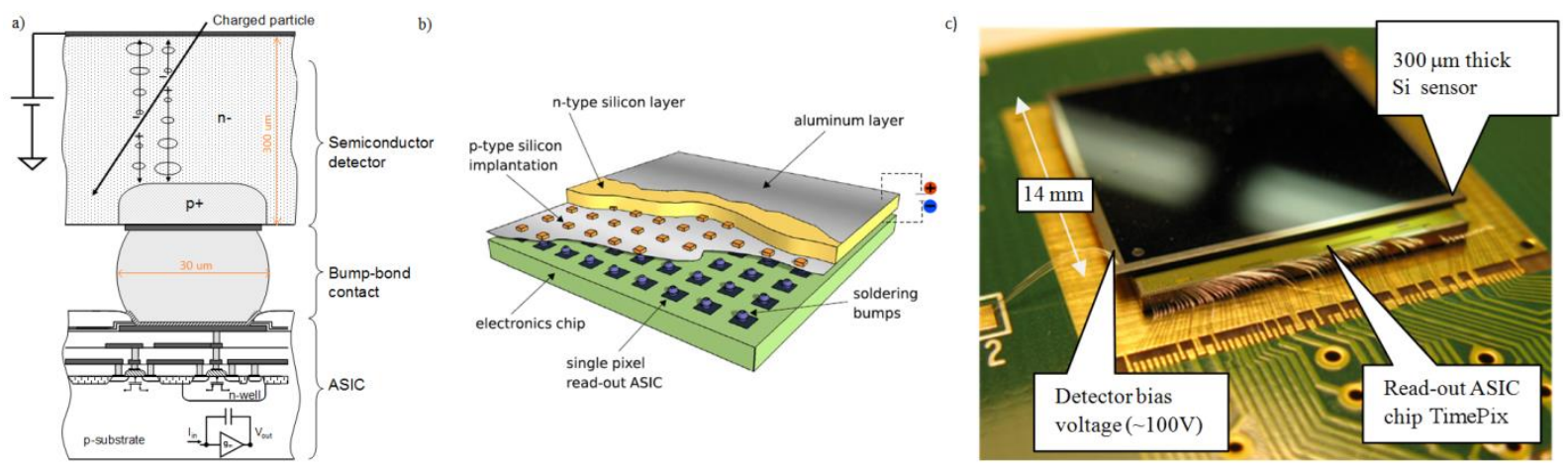

Fig. 1: Illustration (a) at the pixel scale (pixel pitch size $55 \mu \mathrm{m}$ ) of (b) the Timepix detector consisting of a semiconductor sensor (silicon with thickness $300 \mu \mathrm{m}$ ) bump bonded to the integrated ASIC readout chip of $256 \times 256$ pixels (total 65.356 channels). Full detector size (c): $14 \mathrm{~mm} \times 14 \mathrm{~mm}$.

\section{Timepix/SATRAM spacecraft payload}

The Space Application of Timepix based Radiation Monitor (SATRAM) is a technology demonstration payload as a spacecraft platform device on-board ESA's Proba V satellite which was launch on $7^{\text {th }}$ May 2013. The payload contains an FPGA controlling the Timepix detector and provides communication with the spacecraft, along with housekeeping, data compression and configuration. Including the Aluminum alloy compartment the payload has dimensions 108 $\mathrm{mm} \times 63 \mathrm{~mm} \times 56 \mathrm{~mm}$ (see Fig. 2) and total weight $380 \mathrm{~g}$ including the shielding box.
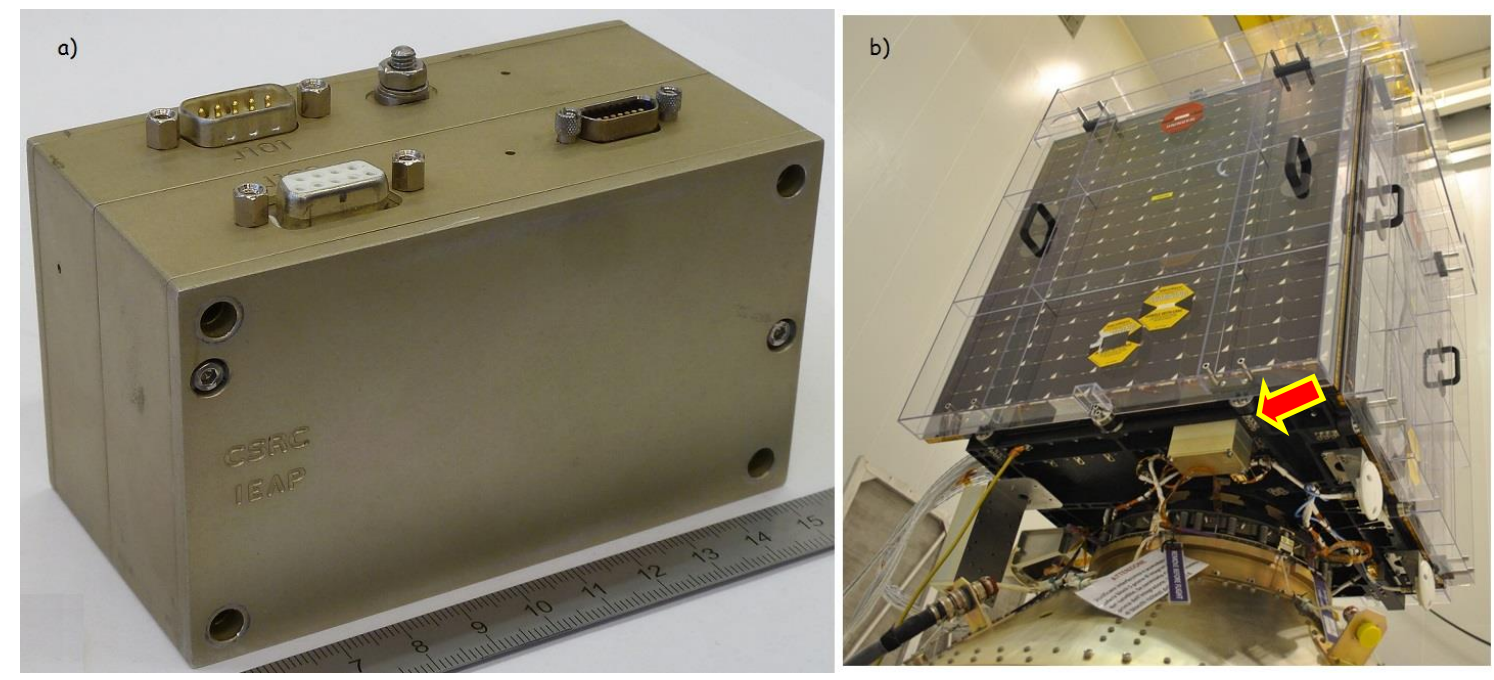

Fig. 2: SATRAM payload (a) attached to ESA's Proba-V satellite (b) prior launch by ESA's Vega-2 rocket. For comparison, Proba-V satellite size: $80 \mathrm{~cm} \times 80 \mathrm{~cm} \times 100 \mathrm{~cm}$ and mass: $138 \mathrm{~kg}$. The plastic side panels with handles are protective covers which were removed before flight.

The entrance window in front of the Timepix chip is of $0.5 \mathrm{~mm}$ thick Al. The payload features $28 \mathrm{~V}$ voltage input and power consumption $\leq 3 \mathrm{~W}$. The energy calibration of the Timepix detector was performed with $\mathrm{X}$-ray and alpha particle radioactive sources. Tests and detector response characterization were done also with a radionuclide neutron source (AmBe) and low- 
energy light ion (proton, deuterium and alpha particle) beams and mono-energetic fast neutron sources from the Van de Graaff accelerator at the IEAP CTU in Prague.

\section{Detection and track visualization of space radiation in open space}

The detection and visualization of space radiation in the form of individual Timepix frames of the radiation environment along the Proba-V satellite orbit are shown in Figs 3-7. The perpixel energy measured is displayed in color in log scale. The frames displayed are labelled according to navigation stamp (geographical position and time) as indicated in Fig. 8. The varying acquisition time of the collected frames (indicated in Figs 3-7) is taken into account in deriving the corresponding particle fluxes (see Sec. 5) as shown in Fig. 8 and Fig. 9. Particle species are distinguished into main types such as light and heavy charged particles. For energetic charged particles also direction is registered (detailed analysis is in progress).

\section{Particle flux along the satellite orbit}

SATRAM can continuously sample the radiation field along the satellite path as shown in Fig. 8 and Fig. 9 where the measured total particle flux is displayed for one orbit (100 minutes) and 19 days (period 1-19 Oct. 2014), respectively. The correlated spatial and time distributions are shown in $\log$ scale. The presented quantity (particle flux) varies up to six orders of magnitude over the high intensity radiation regions (regions near the poles and the South Atlantic Anomaly - SAA).

\section{Conclusions}

SATRAM is the first deployment of the Timepix detector in open space. The spacecraft payload has been successfully commissioned. Detailed data analysis is in progress. The response and track visualization of the varying radiation environment along a single orbit have been presented. The response of the payload with the embedded Timepix detector enables distinguishing the main particle type components present in the radiation environment observed (X-rays, light charged particles and heavy charged particles). Preliminary results have been presented in the form of detection and track visualization of single particles as well as qualitative characterization of the mixed radiation field in the open space environment. Further characterization of the radiation field includes analysis of angle of incidence (for energetic charged particles) and stopping power. Evaluated results include high sensitivity quantum imaging radiation dosimetry and integrated dose rate maps along the satellite orbit. Currently detailed data analysis is in progress including quantitative and time-dependent results on radiation particle composition, particle component fluxes and directional distributions for energetic charged particles. This work extends the use of Timepix into open space started from ground-based [3] and atmospheric/aerospace [7] applications. 

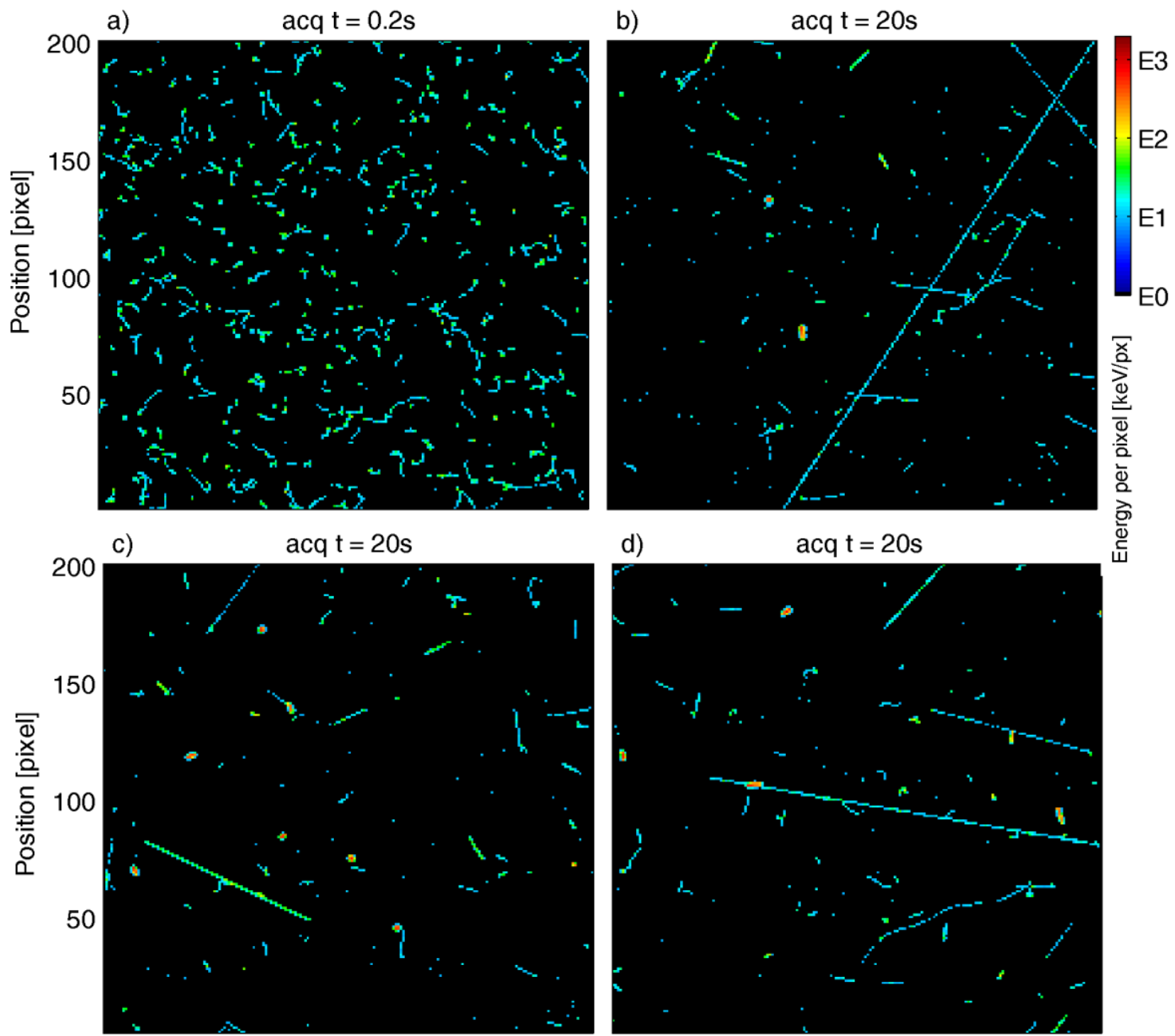

d)

acq $\mathrm{t}=20 \mathrm{~s}$
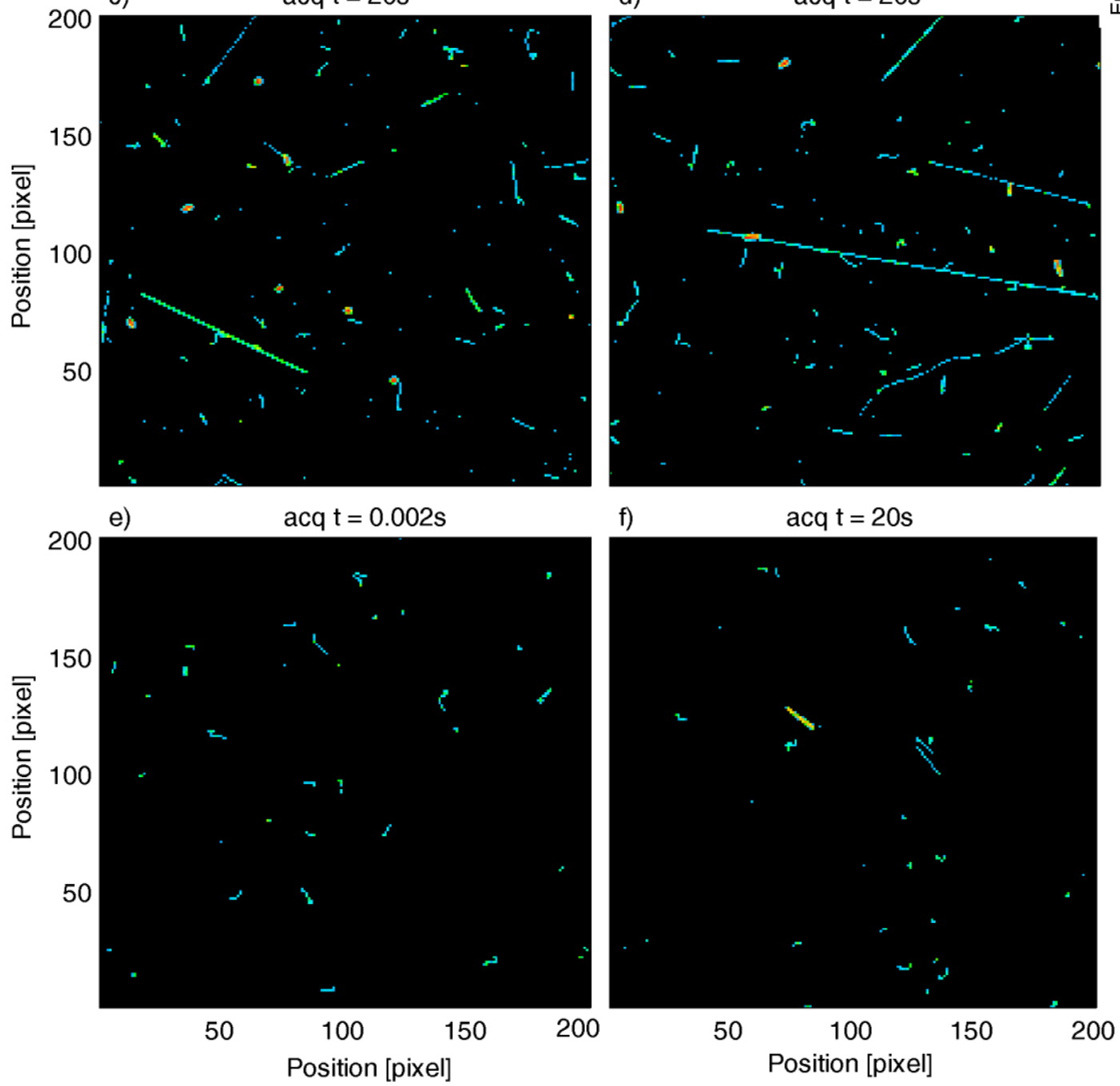

Fig. 3: Quantum imaging detection of space radiation by Timepix/SATRAM payload for selected frames (see labels in Fig. 8 showing the corresponding navigation stamp). Data displayed in Timepix's Timeover-threshold (ToT) mode (per-pixel energy displayed in log scale) with varying acquisition time as indicated. Only part of the sensor matrix $(200 \times 200$ pixels $)$ is displayed $\left(11 \mathrm{~mm} \times 11 \mathrm{~mm}=1.21 \mathrm{~cm}^{2}\right)$. 

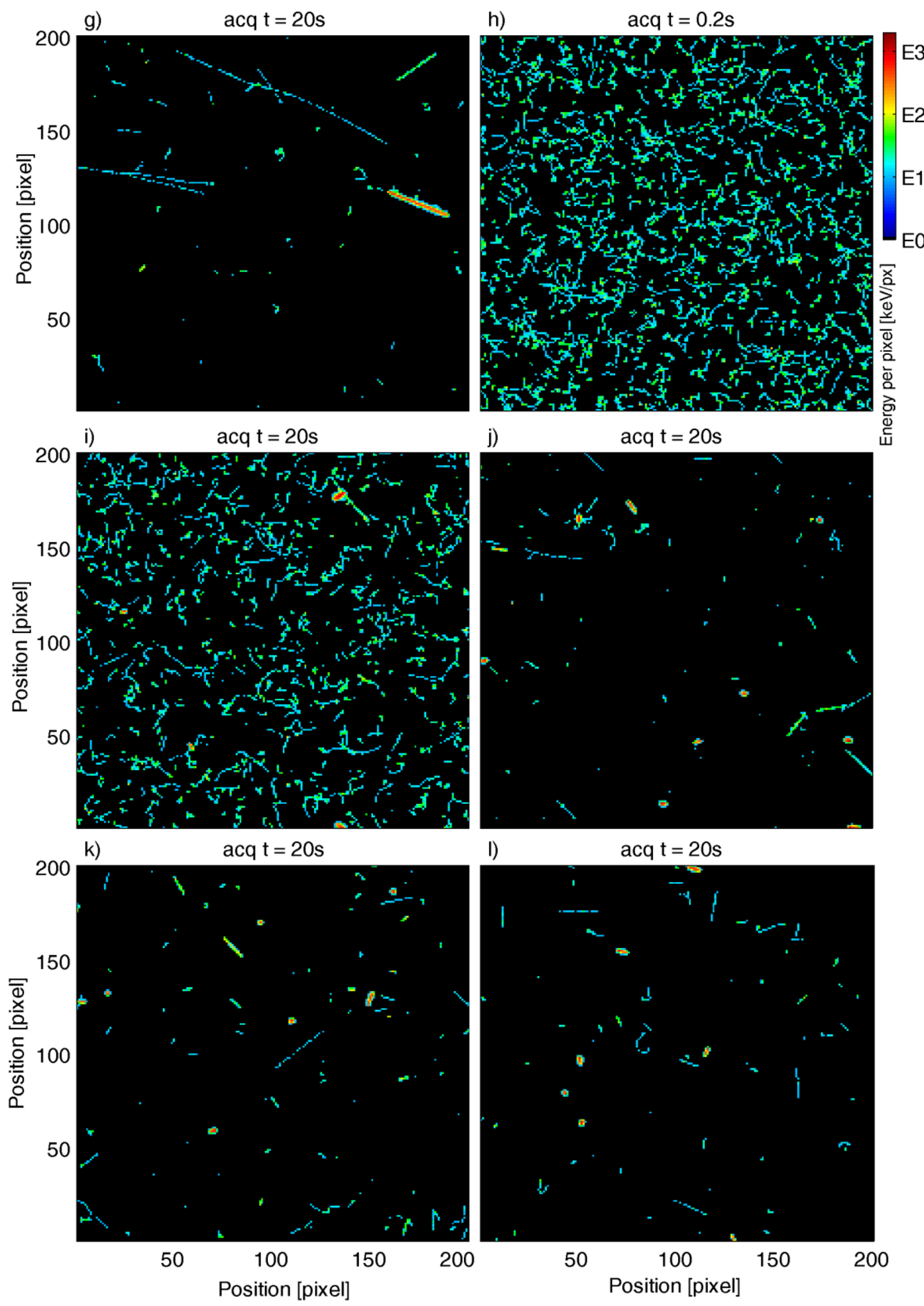

l) $\quad$ acq $t=20 \mathrm{~s}$

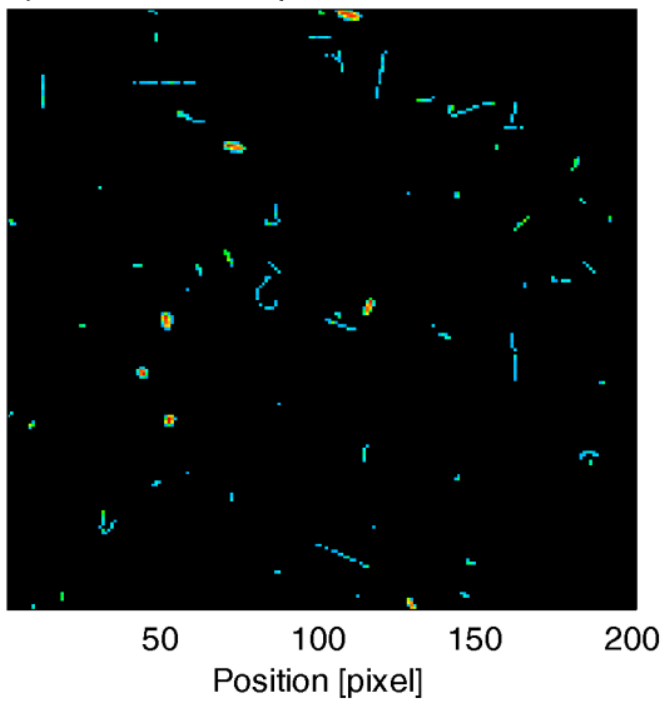

Fig. 4: Same as Fig. 3 - continuation. 

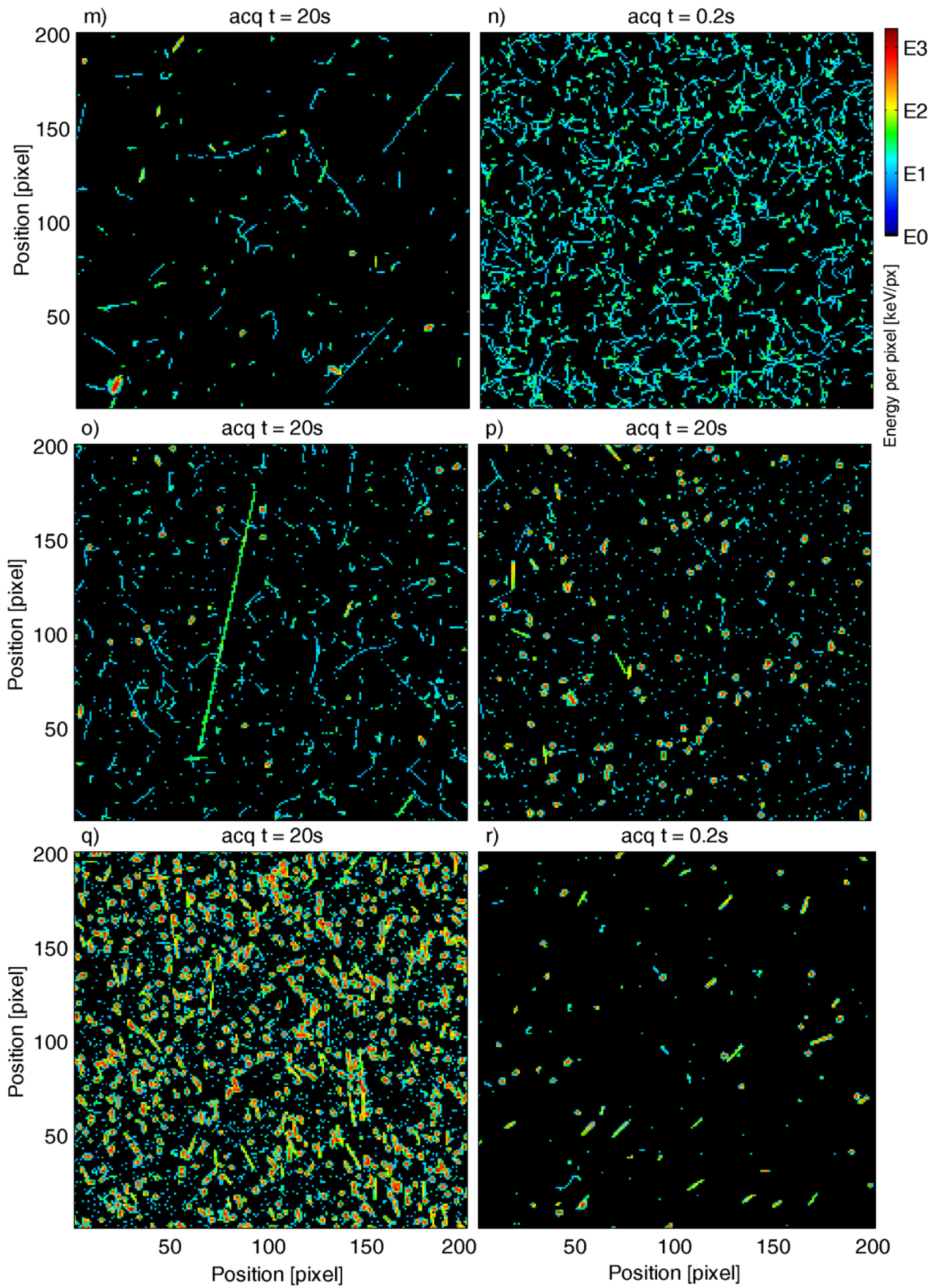

Fig. 5: Same as Fig. 3 - continuation. 

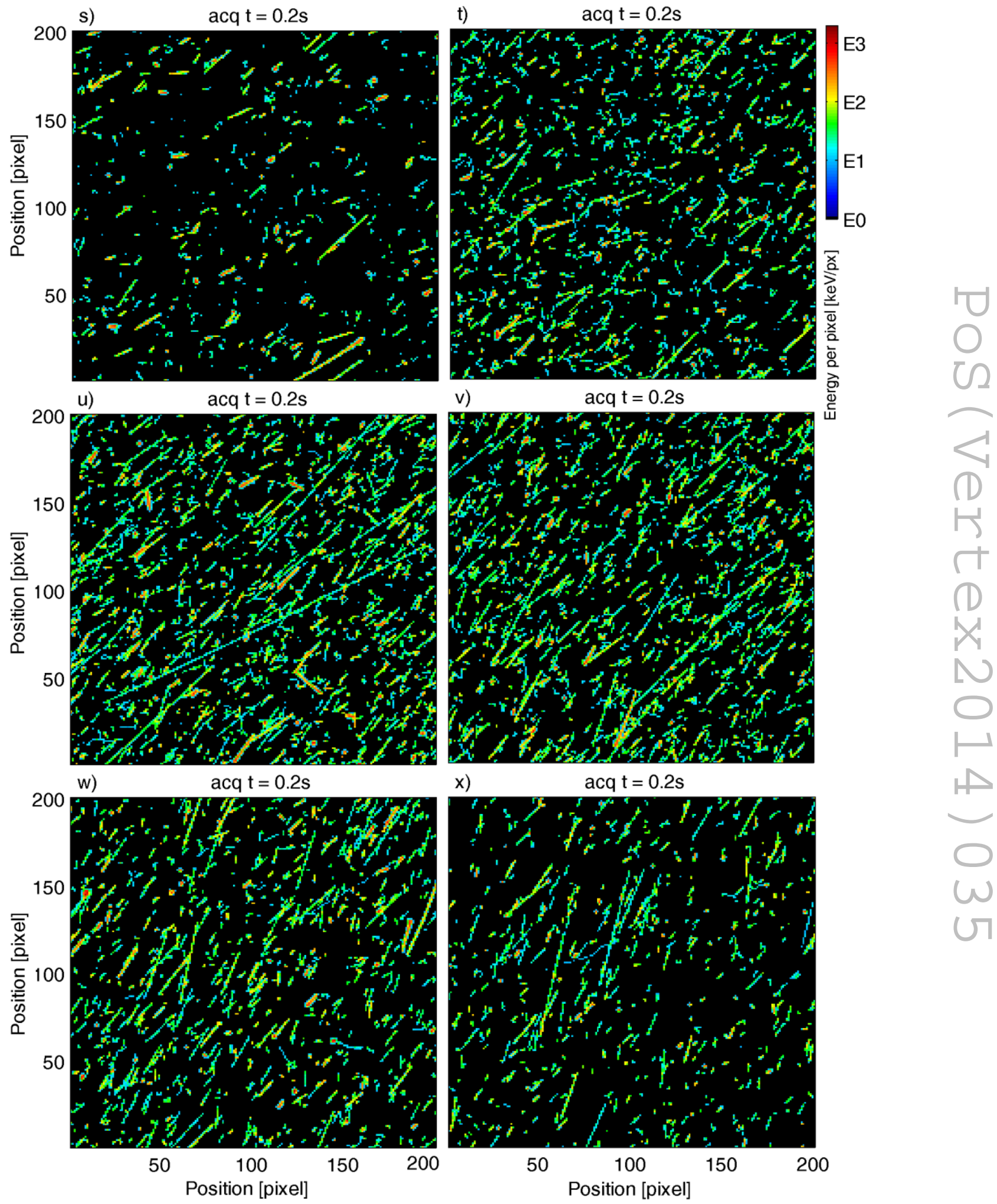

Fig. 6: Same as Fig. 3 - continuation. 

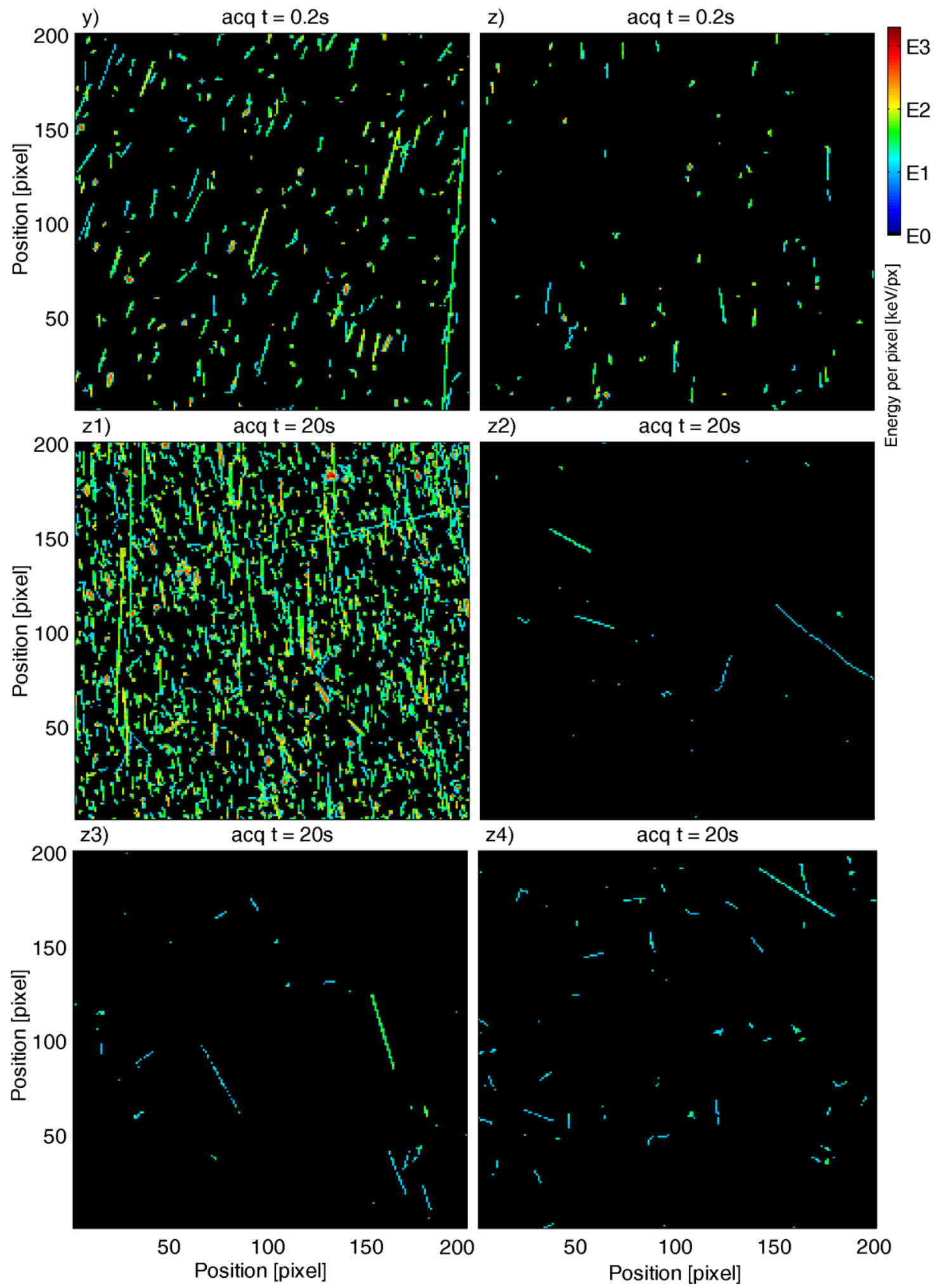

acq $t=20 s$

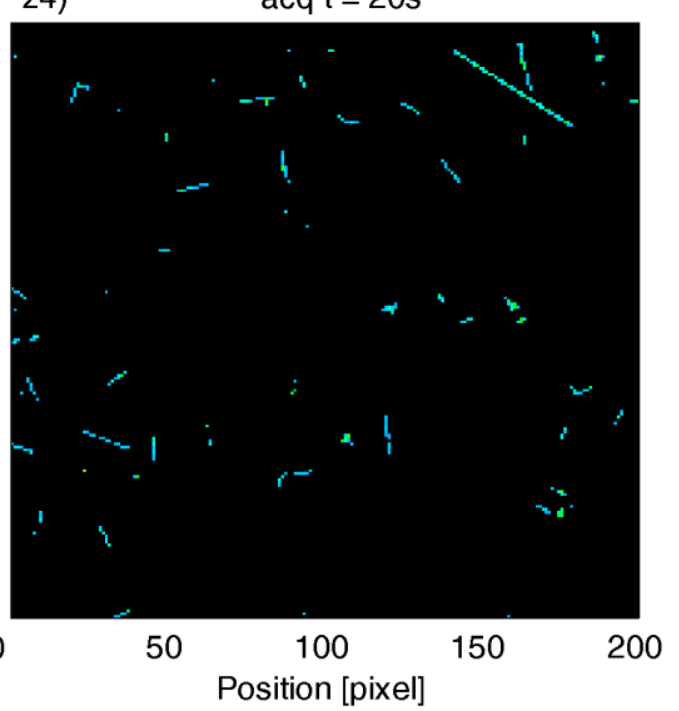

Fig. 7: Same as Fig. 3 - continuation. 


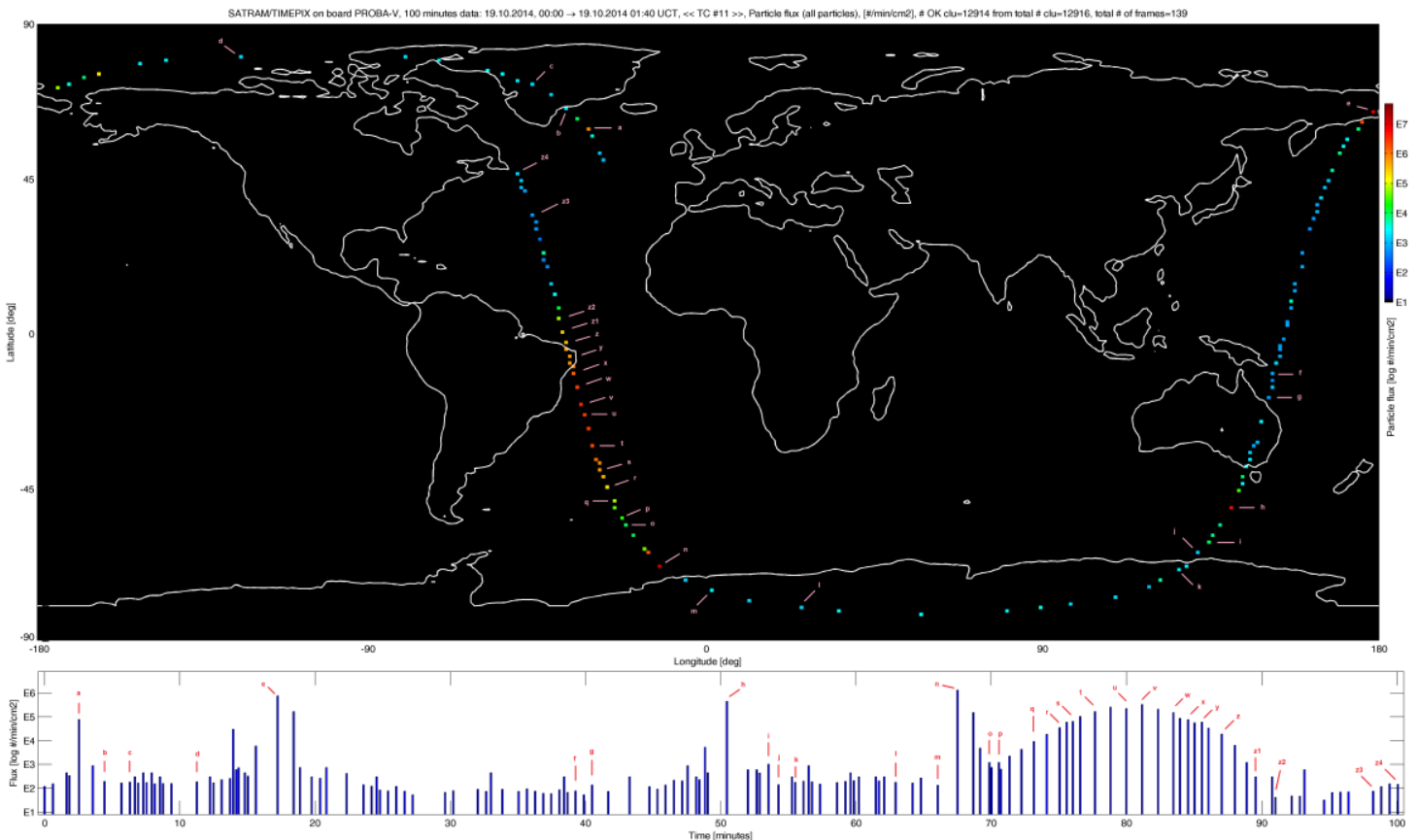

Fig. 8: Flux of all particles registered by SATRAM/Timepix along one orbit of the Proba-V satellite (100 minutes in the period 00:00 thru 01:40 $\mathrm{h}$ on $19^{\text {th }}$ Oct 2014). The spatial (top) and time (bottom) distributions are displayed in log scale. Selected frames (see labels) are displayed in Figs. 3-7.

\section{Acknowledgments}

Research performed in frame of the CERN Medipix Collaboration. Project funded by the European Space Agency, Grant No. 4000105089/11/NL/CBi (641-120004M). Testing and calibration verification measurements of Timepix chips were performed at the Van de Graaff light ion accelerator of the IEAP CTU in Prague in frame of the MSMT Grant Research Infrastructure No. LM2011030 of the Ministry of Education, Youth and Sports of the Czech Republic. The cooperation with Qinetiq operating the Proba-V satellite is gratefully acknowledged.

\section{References}

[1] B. Sean, The mission of Proba-V, ESA Bulletin, European Space Agency 153 (2013) 10-21 (see http://esamultimedia.esa.int/docs/Proba/Bulletin_Proba-v.pdf).

[2] X. Llopart, M. Campbell, E. Heijne, et al., Timepix, a 65 k programmable pixel readout chip for arrival time, energy and/or photon counting measurements, Nucl. Instr. and Meth. in Phys. Res. A 581 (2007) 485-494.

[3] E.H.M. Heijne, R. Ballabriga, M. Campbell, et al. Measuring radiation environment in LHC or anywhere else, on your computer screen with Medipix, Nucl. Instr. and Methods A 699 (2013) 198204.

[4] Z. Vykydal, J. Bouchami, M. Campbell, et al., The Medipix2-based network for measurement of spectral characteristics and composition of radiation in ATLAS detector, Nucl. Instr. Meth. Phys. Res. A 607 (2009) 35-37. 
[5] C. Granja, S. Polansky, Z. Vykydal, et al., Directional Visualization of Space Radiation Quanta with Timepix/SATRAM Spacecraft Payload on board ESA Proba-V Satellite, Proc. of Science PoS (X LASNPA) 003 (2014).

[6] X. Llopart, M. Campbell, R. Dinapoli, et al., Medipix2: a 65-k pixel readout chip with 55 um square elements working in single photon counting mode, IEEE Trans. Nucl. Sci. 49 (2002) 2279-2283.

[7] C. Granja, S. Pospisil, Quantum dosimetry and online visualization of X-ray and charged particle radiation in aircraft at flight altitudes with the pixel detector Timepix, Adv. Space Res. 54 (2014) 241-251. 

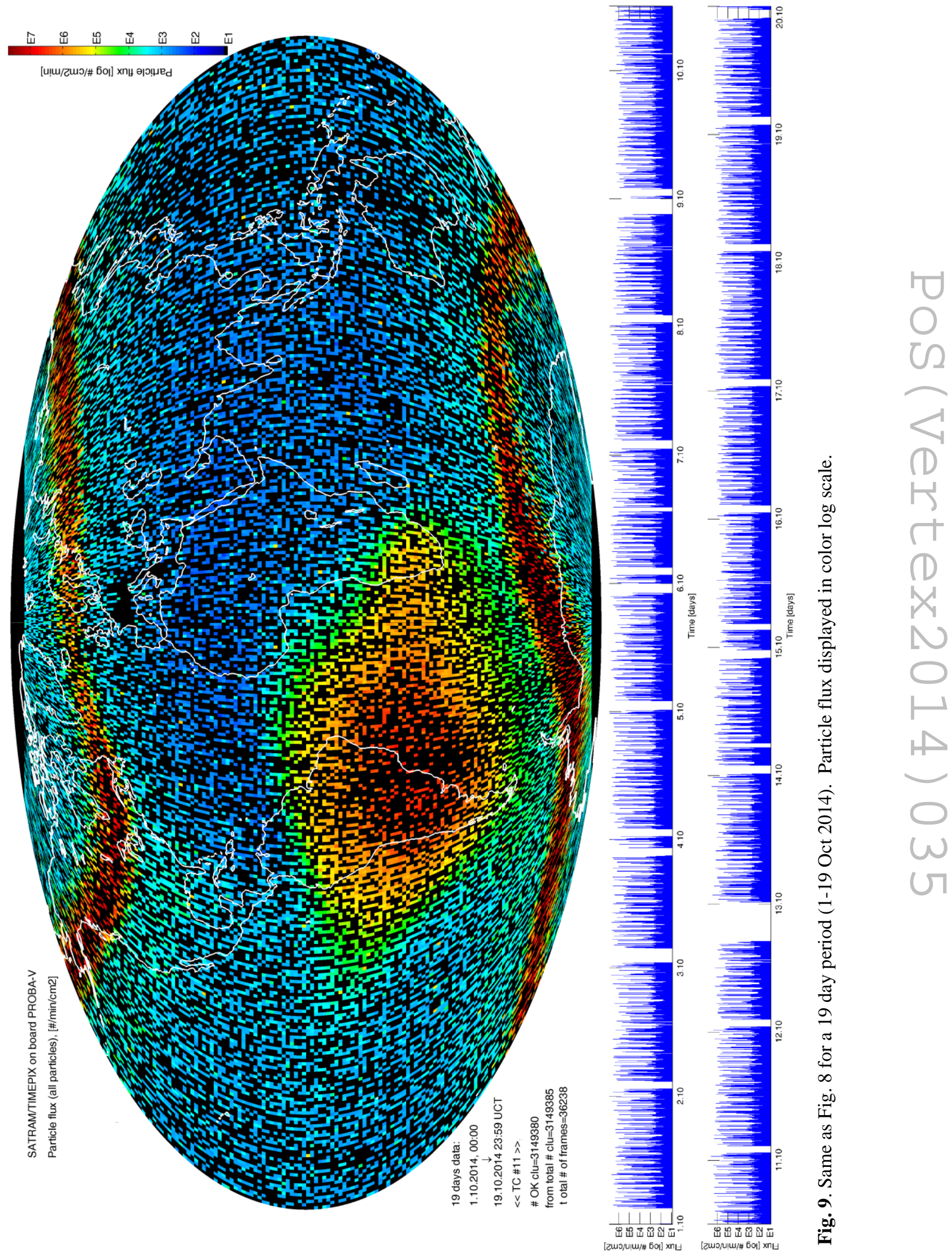